\section{Incidence of Malaria in Tropical Africa}

THE conventional 'parasite-rate' (percentage proportion of children aged 3-10 years in whose blood malaria parasites are detected by a single examination) may be a misleading standard of qualitative measurement giving a distorted idea of the amount of malaria in a locality. But in practice a reduction from 70 to 40 per cent is often claimed to be evidence of effective malaria control, and we are led to believe that a figure of 5 per cent is a splendid achievement indicating a very low malaria incidence.

It has been pointed out that the rate at which infants acquire their first infection offers an exact and quantitative alternative method of measurement. If we can find the number of infective mosquito bites received by people in a given time, we have in a single figure not only the malaria-transmitting ability of the mosquitoes but also the actual incidence of the disease itself ${ }^{1}$.

Working in Freetown, West Africa, we investigated the blood of infants at monthly intervals, and the examinations of fifty individuals made sequentially for eighteen months showed that twelve infections were received during that time. We could say that mosquitoes were infecting one in six infants each year.

Now if we visualize a situation in which one in every six persons in a city in the British Isles were to be infected with malaria each year, the problem of dealing with some 13,000 cases among a population of 80,000 such as existed in Freetown would indeed be a formidable one ! (We are not, however, necessarily inferring that the African may react to infection as severely as the European.) Yet when we came to express the number of examinations of infants in whom malaria parasites were detectable as a percentage of the total examinations made, a parasiterate of 5 per cent was obtained. On the face of it, that is a very small figure and could have easily misled us into a false assumption that the malaria incidence was very low.

Now while the infants were being investigated, mosquitoes were collected from houses in Freetown by standard methods, and the proportion carrying salivary gland infections was measured. From this data it was possible to calculate the 'parasite-rate' of the infants. The accuracy of these estimates encouraged us to calculate theoretical 'parasite-rates' produced by a wide range of numbers of 'infective mosquito bites' received by the average child in a year (see table). These values were in close agreement with observed data.

\begin{tabular}{|l|c|c|c|c|c|c|c|c|c|}
\hline $\begin{array}{l}\text { Number of in- } \\
\text { fective mosquito } \\
\text { bites per child } \\
\text { per year }\end{array}$ & $0 \cdot 01$ & $0 \cdot 1$ & $0 \cdot 2$ & $0 \cdot 4$ & $1 \cdot 0$ & $2 \cdot 0$ & 5 & 10 & 50 \\
\hline $\begin{array}{l}\text { Calculated para- } \\
\text { site-rate \% in } \\
\text { children }\end{array}$ & $0 \cdot 26$ & $2 \cdot 6$ & $5 \cdot 0$ & 10 & 23 & 40 & 73 & $92 \cdot 5$ & $99 \cdot 9$ \\
\hline
\end{tabular}

A reduction of the parasite-rate to 40 from 70 per cent then merely represents the difference between the average child receiving two infections of malaria each year and five. A parasite-rate of 2.5 per cent is revealed as the result of infection of one in ten children each year, which is certainly still too high.

Yet after four years of the most intensive mosquito control in Freetown, it appears that the parasite-rate of school children could not be reduced below a figure of 10 per cent (we can stop mosquitoes breeding but not people moving into and out of our control area). While that is a very low rate for tropical Africa, our investigation suggests that infections are still received by no less than half the children each year.

We feel that were it possible to achieve a situation where one in a thousand persons contract the disease per year, then we might be entitled to say that control of malaria could be effected. But over nearly all tropical Africa the average African seems normally to receive not less than five and frequently many more than fifty infective mosquito bites each year.

Above a figure of 75 per cent the parasite-rate has no practical significance, since the average person is receiving superfluous infections. At satisfactory numbers of infective mosquito bites per year, the parasite-rate is less than 1.0 per cent. Consequently, changes in intervening values have also little practical meaning.

If this be the case, most efforts at malaria control may merely reduce the frequency of receiving infection at the cost of partial loss of immunity without necessarily leading to any appreciable reduction of the disease.

It all depends upon whether we are concerned in protecting small groups of completely non-immune Europeans, or attempting to protect the otherwise immune or partially immune indigenous population. If the latter, it may well be that we shall have to revise our conventional methods of assessing the incidence of malaria, and attempt to discover just how often the average African is, in fact, infected and what exactly are the effects of malaria upon his health and efficiency.

\section{G. A. Walton \\ (Colonial Medical Research Service)}

Department of Entomology,

London School of Hygiene

and Tropical Medicine,

Keppel Street, W.C.1. April 7.

${ }^{1}$ Ann. Trop. Med. Parasit., 41, 380.

\section{Inhibition of Aerobic Sporing Bacilli by Hæmatin}

Certarn bacilli will not grow on nutrient agar containing hæmatin at a concentration of $1 / 20,000$. Other bacilli do not appsar to be affected by hæmatin. The organisms tested are shown in the following table, the figures in brackets being the number of strains of each species tested.

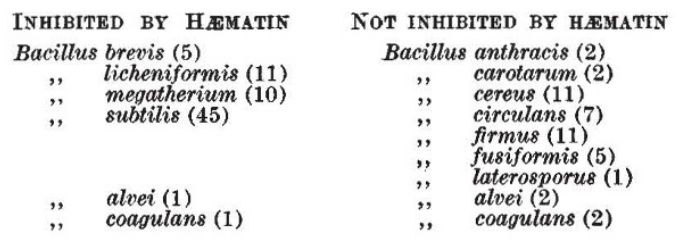

With the exceptions of $B$. alvei and $B$. coagulans, all the tested strains of a given species reacted uniformly towards hæmatin. The growth of $B$. subtilis was completely inhibited by hæmatin at a concentration of $1 / 125,000$, and was partially inhibited by hæmatin at a concentration of $1 / 500,000$. 